\title{
UN ESTUDIO COMPARADO DE LAS ACTITUDES HACIA LA ESTADÍSTICA EN PROFESORES EN FORMACIÓN Y EN EJERCICIO
}

\author{
Estrada, Assumpta ${ }^{1}$; Batanero, Carmen ${ }^{2}$ y Fortuny, Josep Maria ${ }^{3}$ \\ ${ }^{1}$ Departament de Matemàtica. Facultat de Ciències de l'Educació. Universitat de Lleida \\ ${ }^{2}$ Departamento de Didáctica de la Matemática. Facultad de Ciencias de la Educación. Universidad de Granada \\ ${ }^{3}$ Departament de Didàctica de la Matemàtica i les Ciències Experimentals. Universitat Autònoma de Barcelona \\ aestrada@matematica.udl.es \\ batanero@ugr.es \\ JosepMaria.Fortuny@uab.es
}

\begin{abstract}
Resumen. En este trabajo presentamos los resultados de una escala de actitudes en una muestra de 66 profesores en ejercicio y 74 profesores en formación de educación infantil y primaria. No encontramos diferencias por géneros, aunque sí una mejoría en aquellos alumnos que estudiaron estadística previamente. La actitud es algo mejor en los profesores en ejercicio, aunque tiende a empeorar con el uso o la enseñanza de la estadística. Pensamos que estos resultados sugieren la dificultad que tiene dicha materia para los profesores y la necesidad de apoyo que éstos deberían recibir desde los departamentos de didáctica y las facultades de educación.
\end{abstract}

Palabras clave. Actitudes, educación estadística, formación de profesores, didáctica de la matemática, dominio afectivo.

Summary. In this paper we present the results from an attitude scale from a sample of sixty-six in-service teachers and seventy-four trainee primary teachers. Although there were no significant differences by gender, those individuals with previous instruction in statistics performed better. At the same time, attitude was better among in-service teachers; however, attitudes tend to deteriorate with everyday statistics teaching practice. This suggests that statistics is a difficult topic for teachers and that they need extra support from mathematics education departments.

Keywords. Teacher attitudes, statistics education, teacher instruction, mathematics teaching, affective domain.

\section{INTRODUCCIÓN}

En la actualidad, asistimos a un interés creciente hacia la problemática planteada por la formación de profesores de matemáticas, debido, entre otras razones, al fracaso escolar, a la insatisfacción consecuente de los profesores y a las nuevas reformas curriculares, que exigen un reciclaje del profesor en ciertas materias.

Una formación del profesor exclusivamente matemática o psicopedagógica de índole generalista no parece suficiente.
La investigación didáctica centrada en la formación de profesores está produciendo abundante información sobre lo que podemos describir como «conocimiento didáctico del contenido» (Thompson, 1992). Además, el conocimiento profesional implica, según Llinares (1994), el diseño de entornos de aprendizaje, entendiendo como tales, los lugares en los que se pueden originar los problemas a investigar y que deben ayudar a los profesores en formación a cuestionar sus creencias y actitudes previas. 
La estadística es, según Estrada (2001), una materia frecuentemente olvidada por el profesor en la enseñanza obligatoria, a pesar de su utilidad reconocida y de figurar en las directrices curriculares. Para Gattuso y Pannone (2002), una posible explicación es la escasa preparación en la disciplina con la que el profesor termina sus estudios, lo que hace que cuente con pocos recursos para su enseñanza. Esto es un círculo vicioso, puesto que, al no impartir estadística, el profesor no llega a completar sus conocimientos a partir de la práctica docente y las actitudes hacia la materia podrían ser muy similares en los profesores en formación y profesores en ejercicio.

En Estrada (2002) sugerimos la posibilidad de que la actitud hacia la estadística del profesor en ejercicio se deteriore con la práctica docente, debido a la dificultad que él mismo encuentra en la materia, a la escasa importancia que se le otorga, por ejemplo, en los antiguos exámenes de selectividad o a la dificultad para aprender que aprecia en sus alumnos.

Esta actitud negativa de los profesores hacia la estadística podría condicionar la enseñanza y repercutir en las futuras actitudes de sus alumnos, por lo que será necesaria una labor de motivación profunda si queremos que la enseñanza de la estadística sea una realidad y no simplemente un deseo expresado en las orientaciones curriculares.

En este trabajo presentamos un estudio en el que comparamos las actitudes de los profesores en formación con las de los profesores en ejercicio y evaluamos el efecto de diferentes variables sobre ellas. La investigación llevada a cabo se inscribe dentro de un proyecto más amplio, en el campo de la educación estadística, orientado al estudio de las actitudes y conocimientos estadísticos de los profesores (Estrada, 2002) y cuyo objetivo final es fundamentar la acción didáctica que permita incidir en las actitudes de los profesores e indirectamente en la mejora de la enseñanza de la estadística en la educación primaria.

\section{LAS ACTITUDES HACIA LA ESTADÍSTICA Y SUS COMPONENTES}

Existen diversas concepciones respecto al significado del término actitud, y en Auzmendi (1992, p. 16) se analizan algunas de sus definiciones clásicas.

Rokeach (1968, p. 112) las define como «una organización de creencias relativamente permanentes que predisponen a responder de un modo preferencial ante un objeto o situación».

McLeod (1992), al conceptualizar el dominio afectivo de la educación matemática, distingue entre «emociones», «actitudes»y «creencias». Las emociones son respuestas inmediatas, positivas o negativas, producidas mientras se estudian matemáticas o estadística, mientras que las actitudes son respuestas relativamente más estables o sentimientos más intensos que se desarrollan por repetición de respuestas emocionales y se automatizan con el tiempo. Los pensamientos o creencias, en cambio, son las ideas individuales mantenidas durante un largo tiempo que se tienen sobre la materia, sobre uno mismo como estudiante o sobre el contexto social en el que se realiza el aprendizaje.

En la misma línea, Gómez Chacón (2000) entiende «la actitud» como uno de los descriptores básicos del dominio afectivo, junto con «los sentimientos» y «las creencias», y las define como «una predisposición evaluativa (es decir positiva o negativa) que determina las intenciones personales e influye en el comportamiento» (p. 23).

Aplicando esta misma terminología a la educación estadística, Gal, Ginsburg y Schau (1997) indican que durante mucho tiempo los términos actitud y sentimiento han sido utilizados indistintamente. Añaden que, si son intensos, algunos pensamientos o creencias pueden ser el origen del desarrollo de las actitudes hacia la estadística. Entre ellos resaltan los siguientes pensamientos:

- Respecto a la materia (fácil o difícil, requiere habilidades, puede ser dominada por cualquiera).

- Sobre si son una parte de las matemáticas o si simplemente requieren habilidades matemáticas («la estadística es todo cálculo»).

- Sobre el clima del aula y la práctica docente (los ejemplos son extraídos del mundo real, no de libro).

- Sobre uno mismo, sobre cómo se aprende estadística o matemáticas («no sé nada de la materia», «soy bueno en esto»).

- Sobre la utilidad o valor de la matemática y su importancia en su futuro profesional («nunca utilizaré esta materia», «no sirve para nada»).

Según Auzmendi (1992), las actitudes hacia las matemáticas y la estadística surgen en edades muy tempranas y, si bien suelen ser favorables en un principio, tienden a evolucionar negativamente (Suydam, 1984) y persisten con el paso del tiempo (Aiken, 1974). Auzmendi (1992), Gil Flores (1999) y Gómez Chacón (2000) diferencian en ellas tres factores básicos, llamados también componentes pedagógicos:

- Componente cognitivo: Se refiere a las expresiones de pensamiento, concepciones y creencias, acerca del objeto actitudinal, en este caso, la estadística. Incluye desde los procesos perceptivos simples, hasta los cognitivos más complejos.

- Componente afectivo o emocional: Está constituido por expresiones de sentimiento hacia el objeto de referencia. Recogería todas aquellas emociones y sentimientos que despierta la estadística, y por ello son reacciones subjetivas positivas/negativas, acercamiento/huida, placer/dolor.

- Componente conductual o tendencial: Aparece vinculado a las actuaciones en relación con el objeto de las actitudes. Son expresiones de acción o intención conduc- 
tista o conductual y representan la tendencia a resolverse en la acción de una manera determinada.

En nuestro estudio tenemos en cuenta estos componentes cognitivo, afectivo y conductual, así como los componentes llamados antropológicos, a saber:

- Componente social: Relacionado con la percepción y la valoración del papel de la estadística en el ámbito sociocultural de cualquier ciudadano.

- Componente educativo: Vinculado al interés hacia la estadística y su aprendizaje, a la visión de su utilidad para el alumno, a su opinión sobre si debiese incluirse en el currículo y a la dificultad percibida.

- Componente instrumental: Referido a la utilidad en otras materias, como forma de razonamiento y como componente cultural.

En esta investigación, y después de analizar diferentes consideraciones sobre la naturaleza de las actitudes hacia la estadística, nos situamos en la línea de Gal, Ginsburg y Schau (1997) y las definimos como:

«Una suma de emociones y sentimientos que se experimentan durante el período de aprendizaje de la materia objeto de estudio» (p. 40).

Además tal como podemos ver en Estrada (2001), los estudios multidimensionales sobre las actitudes son ahora los más utilizados. Por ello, partimos de un concepto pluridimensional de las actitudes hacia la estadística de los profesores en formación y en ejercicio y estructuramos nuestra escala de medición según los componentes pedagógicos y antropológicos definidos anteriormente.

\section{Investigaciones previas}

Las investigaciones realizadas sobre actitudes hacia la estadística se han centrado, fundamentalmente, en la construcción de escalas de evaluación. Así la Statistics Attitude Survey (SAS) elaborada por Roberts y Bilderback (1980) para suplir la necesidad de medir las actitudes de sus estudiantes; y el ATS de Wise (1985), cuyo objetivo principal era la medida del cambio actitudinal al finalizar un curso de estadística básica.

Auzmendi (1992), además de construir su propia escala, va más allá y analiza en su trabajo los factores que constituyen las actitudes hacia las matemáticas y la estadística, así como su vinculación con el logro en estudiantes matriculados en la asignatura de estadística de todas las carreras de la Universidad del País Vasco en las que se impartía algún curso de iniciación a la materia.

Otro trabajo que centra su estudio en la consideración multidimensional de las actitudes hacia la estadística es el de Schau y otros (1995). Al igual que en el caso de Auzmendi, analizan diferentes escalas de medición de actitudes hacia la estadística y, al constantar que no cumplen con una serie de características, deciden construir una nueva escala, la SATS, con dos versiones (pre y post) del cuestionario, realizadas antes y después de recibir la instrucción.

Finalmente tenemos la Escala de atitudes em relaçao à Estatística de Cazorla y otros (1998), escala unidimensional compuesta por 20 ítems y que resulta de la adaptación de la escala de actitudes hacia las matemáticas de Aiken (1974).

Otras se han realizado con alumnos universitarios de diferentes carreras: Gil Flores (1999), con estudiantes de pedagogía y Mastracci (2000), con estudiantes de diferentes especialidades universitarias, todos ellos siguiendo un curso de estadística.

No hemos encontrado estudios, en la bibliografía revisada, que se lleven a cabo específicamente con profesores. Wilensky $(1995,1997)$ describe uno de los pocos trabajos que se centran en el efecto producido por una enseñanza específica en la mejora de las actitudes hacia la estadística, aunque su estudio se realiza sólo con unos pocos casos y se preocupa exclusivamente por el factor ansiedad.

Finalmente, indicamos que Gal, Ginsburg y Schau (1997), conscientes de que las actitudes y creencias de los alumnos pueden interferir, bien dificultando o favoreciendo el aprendizaje, así como afectar en el momento de aplicar estos conocimientos fuera del aula, dedican un capítulo del libro The Assessment Challenge in Statistics Education para alertar a los profesores de la importancia de valorar las actitudes rigurosamente. Para ello describen y analizan diferentes métodos desarrollados para valorarlas, proporcionan sugerencias de utilización y aplicación, así como futuras líneas de investigación.

\section{METODOLOGÍA}

A continuación concretamos el estudio a realizar a partir del establecimiento de hipótesis y variables.

Hipótesis 1. Las actitudes hacia la estadística en profesores en formación y en profesores en ejercicio tiene características muy similares. Puesto que la estadística no se imparte, o se imparte muy someramente, durante la enseñanza obligatoria, los profesores en ejercicio no tienen un contacto profesional con la estadística, o lo tienen escasamente, que les permita modificar las actitudes que tenían como profesores en formación.

Hipótesis 2. La actitud de los profesores en formación y la de los profesores en ejercicio están relacionadas significativamente con ciertas variables personales y escolares: género, especialidad estudiada, nivel escolar en que estudió estadística, años de docencia en general y en la materia y uso de la estadística como instrumento.

La actitud hacia la estadística de profesores en ejercicio y en formación es la variable dependiente de nuestro 
estudio y será operativizada por medio de la puntuación total obtenida en una escala de actitudes, que describimos posteriormente.

Como variables independientes consideramos ciertas variables personales y escolares: género, especialidad y nivel escolar en que estudió estadística para todo el colectivo; años de docencia en general y en la materia para los profesores en ejercicio y uso en clase de la estadística como herramienta multidisciplinar, sólo para los profesores en ejercicio del área de sociales.

\section{Instrumento para la toma de datos y su construcción}

Una vez planteadas las hipótesis y delimitadas las variables, nos dedicamos a reflexionar sobre el instrumento de medida adecuado y elegimos, entre todas las técnicas de observación existentes, la escala de actitud, debido a las razones contempladas en Gairín (1987).

En el momento de llevar a cabo nuestro estudio, no encontramos ninguna escala de actitudes hacia la estadística que contemplara los componentes pedagógicos y antropológicos anteriormente descritos. Por ello se decidió construir un instrumento de evaluación propio combinando las tres escalas más usuales, a saber: escala $S A S$ (Roberts y Bilderback, 1980); escala ATS (Wise, 1985) y la de Auzmendi (1992). En el proceso de elaboración se han seguido las recomendaciones de Osterlind (1989) y Thorndike (1989). En primer lugar, se delimitó el contenido a evaluar y se especificó el formato de los ítems. Éstos constan de un enunciado y una escala de 5 puntos, que valoran las respuestas desde «muy en desacuerdo» (1 punto) hasta «muy de acuerdo» (5 puntos).

A partir de las tres escalas citadas, se elaboró una primera lista de ítems; seguidamente se realizó una selección contemplando los diferentes componentes pedagógicos y antropológicos y, dando un peso equivalente a cada uno, se fue intentando incluir tanto ítems redactados en forma afirmativa ( «la estadística ayuda a entender el mundo de hoy»), como otros en forma negativa («en la escuela no se tendría que enseñar estadística»). Y todo ello para evitar el problema de la aquiescencia (Morales, 1988), por el que algunos sujetos tienden a responder con la forma «de acuerdo» sea cual sea el contenido del ítem.

Tabla I

Componentes de las actitudes evaluadas en la escala.

\begin{tabular}{|l|c|c|c|}
\hline & \multicolumn{3}{|c|}{ Componente antropológico } \\
\hline $\begin{array}{l}\text { Componente } \\
\text { pedagógico }\end{array}$ & Social & Educativo & Instrumental \\
Afectivo & $1,11,25$ & $7,12,23$ & $10,13,16,20$ \\
Cognitivo & $2,19,21$ & $4,6,17$ & 3,24 \\
Comportamental & 9,18 & $8,15,22$ & 5,14 \\
\hline
\end{tabular}

Se consiguió una lista de 36 enunciados, que se sometieron a un «panel de jueces», es decir, expertos con diferentes perfiles profesionales y que emiten su opinión respecto a la adecuación y univocidad de las sentencias, quedando después de la valoración la escala definitiva (Tabla II) compuesta por 25 ítems (14 afirmativos frente a 11 negativos) y cuya distribución según los componentes evaluados por cada ítem es la que aparece en la tabla I.

\section{Muestra participante}

La composición de la muestra intentó reflejar, lo más fielmente posible, los dos grandes colectivos sometidos a estudio, así como también tener en cuenta las variables independientes consideradas. En el caso de los profesores en ejercicio, aprovechamos, para contactar con ellos, un curso de posgrado que realizaban profesores de otro departamento, mientras que los profesores en formación completaron el cuestionario durante una de las sesiones de clase de otra área de conocimiento, para evitar posibles influencias.

La submuestra de profesores en formación está formada por alumnos de magisterio. Elegimos a los de $2^{\circ}$ curso, porque ya empiezan a «sentirse» futuros docentes, puesto que ya han hecho prácticas y han tenido contacto con centros de primaria, aunque aún no han recibido instrucción en el campo de la estadística y su didáctica, hecho que de una u otra forma podría influir en las respuestas.

Hemos elegido para nuestro estudio las especialidades de primaria y de educación infantil porque son las que, en el plan de estudios, presentan una mayor incidencia horaria de los créditos del área de didáctica de la matemática. El grupo resultante está formado por 74 alumnos repartidos entre 36 alumnos de educación primaria y 38 de infantil de la Universitat de Lleida. Son alumnos con las características habituales de nuestra facultad: predominan las mujeres frente a los hombres, las edades son las correspondientes al curso en que se encuentran (20-21 años), el nivel académico es aceptable y en el aspecto social sólo cabe destacar la gran incidencia del medio rural.

La submuestra de los profesores en ejercicio se ha tomado en la provincia de Lleida. Se repartió en dos subgrupos iguales, en cuanto a número y centro donde impartían su docencia. Uno de ellos está formado por maestros del área de ciencias sociales (34), que usan o pueden usar la estadística como instrumento en los últimos cursos de primaria; el otro lo constituyen profesores del área de ciencias (32), que imparten o deberían impartir clases de estadística. Este colectivo de docentes es representativo en cuanto a su composición, en el sentido de abarcar tanto a la escuela pública como la privada, la rural como la urbana.

Hubo una fuerte incidencia de las mujeres, que representan un $79 \%$ del total, algo común en el ámbito de la docencia y, en particular, entre los profesores en formación y en ejercicio en la ciudad de Lleida. Pensamos, en consecuencia, que la muestra, aun siendo intencional, representa a la población estudiada. Nos parece interesante resaltar cómo, a pesar de que oficialmente la estadística 
está presente en los currículos de los diferentes niveles educativos, sólo un $38 \%$ de los profesores en formación la estudió en la EGB y, lo que es peor, un $14 \%$ de ellos llegaron a la universidad sin haberla tratado en toda su vida académica.

Para el colectivo de profesores en ejercicio, un $3 \%$ de los encuestados tiene más de veinticinco años de docencia, un $18 \%$ tienen más de veinte años de docencia y, además, la mediana de la distribución se sitúa alrededor de los diez años. Esto implica una muestra formada por profesores tanto jóvenes (en el sentido de que su instrucción se ha realizado en el mismo plan de estudios de magisterio que los profesores en formación participantes, y es relativamente reciente, ya que más de la mitad, el $54 \%$ exactamente, no supera los quince años de docencia) como experimentados (puesto que el $46 \%$ sí los supera). Respecto a los años de docencia en matemáticas, la media es de 12,1 años, y la distribución es prácticamente normal, situándose la moda en 14 años y la mediana alrededor de los 13 años, por lo que consideramos que, en general, los profesores del área de ciencias evaluados tienen una buena experiencia docente en matemáticas.

Los profesores del área de sociales son los únicos que tienen en sus cuestionarios la pregunta de si utilizan la estadística en sus clases como herramienta de trabajo. De los 34 profesores encuestados, el $65 \%$ la utiliza algunas veces, mientras que un escaso $6 \%$ no la utiliza nunca. Notamos que un $30 \%$ dice que lo hace habitualmente, dato que no está en consonancia con los estudios previos conocidos sobre el tema, ni los indicios que nos indujeron a realizar esta investigación.

\section{ANÁLISIS DE RESULTADOS}

\section{Análisis de resultados por ítems}

En la tabla II presentamos los resultados referentes a cada uno de los 25 ítems, tal como fueron presentados a los sujetos de la muestra. Hacemos constar el número de casos de cada una de las categorías $(1=$ muy en desacuerdo, $2=$ en desacuerdo, $3=$ indiferente, $4=$ de acuerdo, $5=$ muy de acuerdo) para el total de la muestra. Presentamos también las medias y desviaciones típicas de las puntuaciones obtenidas con el criterio anterior. Hacemos notar que los ítems 1, 3, 6, 9, 11, 14, 15, 19, 21, 23 y 25 tienen un enunciado desfavorable a la actitud que tratamos de medir $y$, para poder interpretar sus resultados, es necesario tener en cuenta que una puntuación alta en realidad indica una actitud negativa y, por tanto, en el momento del vaciado de los cuestionarios, se puntuó al contrario $(1=$ muy de acuerdo, $2=$ de acuerdo, $3=$ indiferente, 4 = en desacuerdo, $5=$ muy en desacuerdo).

Esta decisión se toma porque, en el cálculo de la puntuación total, es necesario que todos los ítems tengan la misma dirección y sobre todo para poder tener una escala homogénea de comparación de todos los ítems, en la que una media más (o menos) alta indique siempre una actitud más (o menos) positiva, independientemente de si el ítem se redacta con enunciado positivo o negativo. De esta manera, la puntuación total (suma de las puntuaciones de los 25 ítems) representará la actitud de cada encuestado respecto a la estadística y, ésta será tanto más favorable cuanto más elevada sea esta puntuación.

Las medias y desviaciones típicas se calculan respecto a la puntuación dada en la respuesta y, en consecuencia, siempre se deben interpretar en una escala positiva. Por ejemplo, en el enunciado 6 (En la escuela no se tendría que enseñar estadística), hemos obtenido una media global de 4,13 puntos, que indica una actitud positiva, es decir, que los profesores en formación son contrarios al enunciado del ítem y piensan que en la escuela debería enseñarse la estadística. Podemos ver que en este ítem, efectivamente, la mayoría de los encuestados está en desacuerdo con el enunciado (44 sujetos muy en desacuerdo, que puntúan como $5 ;$ y 76 en desacuerdo, que puntúan como 4).

Entre los ítems mejor valorados, con 4,31 puntos, destacamos el ítem 20 (Me gusta hacer problemas cuando uso la estadística). Corresponde a un componente afectivo, al ser una manifestación de un sentimiento o afecto hacia la materia que, además, en este caso, es positiva. Parece, por las puntuaciones elevadas otorgadas por los profesores, que en esta materia prefieren la resolución de problemas al trabajo teórico. Estas ideas de los profesores están en concordancia con Tanur (1992), que presenta la estadística como una potente herramienta de trabajo multidisciplinar y utilizada además en muy diferentes ámbitos. Con una puntuación ligeramente inferior $(4,29)$, tenemos el ítem 18 (Me entero más del resultado de las elecciones cuando aparecen representaciones gráficas), constatando así la importancia que se otorga a la presencia de la estadística en la vida cotidiana, otro de los pilares básicos que justifican su presencia en la enseñanza obligatoria, como formación básica de todos los ciudadanos (Gal, 2002).

Dadas las características del colectivo estudiado (profesores en formación y en ejercicio), nos parece de interés destacar los otros dos ítems mejor valorados, que son el 6 (En la escuela no se tendría que enseñar estadística), con el que los encuestados estuvieron en desacuerdo; y el 22, (A menudo explico a mis compañeros problemas de estadística que no han entendido), con medias de 4,13 y 4,06 respectivamente. Estos enunciados corresponden a aspectos educacionales que muestran el interés de los profesores por la enseñanza de la estadística y en los que se puede apreciar la conveniencia de tratar estos temas de estadística y probabilidad en los currículos de la enseñanza obligatoria. Esta necesidad evidenciada implica que, para ello, es necesaria una formación específica adecuada. En general, todos los ítems tienen una valoración positiva, puesto que una puntuación 3 indicaría una actitud neutra y prácticamente todos tienen un valor medio superior a 3. Entre los que no alcanzan este valor, el peor de todos es el ítem 23 (Si pudiera eliminar alguna materia, sería la estadística) con 2,49 y sentencia negativa. Esto contrasta algo con los resultados en los ítems anteriores, aunque pudiera indicar una preferencia por otras materias más que un rechazo hacia la estadística. 


\begin{tabular}{|c|c|c|c|c|c|c|c|}
\hline Enunciado del ítem & 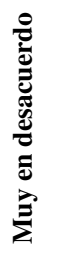 & 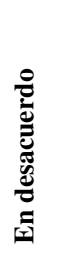 & & 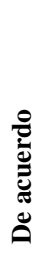 & 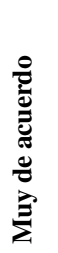 & 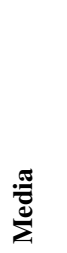 & 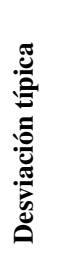 \\
\hline 1. Me molesta la información estadística que aparece en algunos programas de televisión & 10 & 56 & 46 & 24 & 4 & 3,31 & 0,94 \\
\hline 2. La estadística ayuda a entender el mundo de hoy. & 0 & 14 & 22 & 92 & 12 & 3,73 & 0,76 \\
\hline 3. A través de la estadística se puede manipular la realidad. & 10 & 54 & 14 & 40 & 22 & 2,93 & 1,26 \\
\hline 4. Es fundamental en la formación básica del futuro ciudadano. & 2 & 20 & 34 & 70 & 14 & 3,53 & 0,91 \\
\hline 5. Uso la estadística para resolver problemas de la vida cotidiana. & 0 & 22 & 34 & 80 & 4 & 3,47 & 0,79 \\
\hline 6. En la escuela no se tendría que enseñar estadística. & 44 & 76 & 14 & 6 & 0 & 4,13 & 0,76 \\
\hline 7. Me divierto en las clases en que se explica estadística. & 8 & 26 & 76 & 30 & 0 & 2,91 & 0,79 \\
\hline 8. Los problemas de estadística me resultan fáciles. & 6 & 8 & 20 & 78 & 28 & 3,81 & 0,96 \\
\hline 9. No entiendo las informaciones estadísticas que aparecen en la prensa. & 14 & 80 & 26 & 18 & 2 & 3,61 & 0,89 \\
\hline $\begin{array}{l}\text { 10. Me gusta la estadística porque me ayuda a comprender más profundamente la complejidad de } \\
\text { ciertos temas. }\end{array}$ & 2 & 8 & 36 & 70 & 24 & 3,76 & 0,86 \\
\hline 11. Me siento intimidado ante datos estadísticos. & 16 & 80 & 38 & 4 & 2 & 3,74 & 0,75 \\
\hline 12. Encuentro interesante el mundo de la estadística. & 6 & 44 & 20 & 52 & 18 & 3,23 & 1,15 \\
\hline 13. Me gustan los trabajos serios en que aparecen estudios estadísticos. & 2 & 20 & 14 & 76 & 28 & 3,77 & 0,98 \\
\hline 14. Utilizo poco la estadística fuera de la escuela. & 22 & 76 & 38 & 4 & 0 & 3,83 & 0,72 \\
\hline 15. En clase de estadística nunca entiendo de qué están hablando. & 12 & 84 & 24 & 18 & 2 & 3,61 & 0,87 \\
\hline 16. Me apasiona la estadística porque ayuda a ver los problemas objetivamente. & 4 & 40 & 56 & 34 & 6 & 2,99 & 0,91 \\
\hline 17. La estadística es fácil. & 4 & 10 & 26 & 58 & 42 & 3,89 & 1,01 \\
\hline 18. Me entero más del resultado de las elecciones cuando aparecen representaciones gráficas. & 2 & 0 & 0 & 92 & 46 & 4,29 & 0,62 \\
\hline 19. La estadística sólo sirve a la gente de ciencias. & 2 & 18 & 86 & 28 & 6 & 2,87 & 0,74 \\
\hline 20. Me gusta hacer problemas cuando uso la estadística. & 0 & 2 & 10 & 70 & 58 & 4,31 & 0,67 \\
\hline 21. La estadística no sirve para nada. & 2 & 12 & 82 & 36 & 8 & 2,74 & 0,75 \\
\hline 22. A menudo explico a mis compañeros problemas de estadística que no han entendido. & 0 & 4 & 30 & 60 & 46 & 4,06 & 0,81 \\
\hline 23. Si pudiera eliminar alguna materia, sería la estadística. & 0 & 10 & 54 & 70 & 6 & 2,49 & 0,69 \\
\hline 24. La estadística ayuda a tomar decisiones más documentadas. & 2 & 12 & 30 & 72 & 24 & 3,74 & 0,89 \\
\hline 25. Evito las informaciones estadísticas cuando las leo. & 42 & 70 & 20 & 6 & 2 & 4,03 & 0,86 \\
\hline
\end{tabular}

Otros ítems con puntuaciones por debajo de 3 puntos son el ítem 3 (A través de la estadística se puede manipular la realidad), que sugiere desconfianza hacia los datos estadísticos, en coincidencia con numerosos autores, que señalan que la estadística cuenta a veces con mala imagen, debido al abuso que de ella se hace en publicidad o en política.

Las respuestas con baja puntuación en los ítems 7 (Me divierto en clase de estadística) y 16 (Me apasiona la estadística), sugieren que la estadística resulta una materia aburrida. Esto concuerda con lo especificado por Moore (1997) y no es en realidad una falta de la propia disciplina, sino de la manera en que se enseña. Habría que seguir las recomendaciones del autor citado, quien sugiere, por un lado, cambiar los contenidos, enseñando una estadística basada en los datos, con menor énfasis en la probabilidad, que resulta más difícil a los alumnos; y, por el otro, cambiar la metodología, introduciendo la tecnología y el trabajo con proyectos.

Los ítems 19 (La estadística sólo sirve a la gente de ciencias) y 21 (La estadística no sirve para nada) indican que los profesores no llegan a percibir completamente la utilidad de esta materia y no aprecian su utilidad en su propia labor profesional o en las ciencias sociales. 
Una vez analizados los resultados por ítem, hicimos un estudio de fiabilidad por medio del programa SPSS, obteniendo un valor del coeficiente de consistencia interna, $\alpha$ de Cronbach, de 0,774 , a nuestro entender suficiente para el estudio, teniendo en cuenta el tamaño restringido de la muestra.

\section{Resultados globales}

En la figura 1 presentamos el histograma de frecuencias de la puntuación total en la escala de actitudes hacia la estadística para el total de la muestra. A la vista de los resultados obtenidos, y teniendo en cuenta que la puntuación correspondiente a la posición de indiferencia es 75 , podemos afirmar que la actitud de los encuestados respecto a la estadística es positiva, ya que solamente 8 individuos obtienen puntuaciones inferiores y la gran mayoría se concentra en una actitud positiva alrededor de 91 (moda). Es también alto el valor de la media $(88,8)$ y podemos observar en el histograma la forma aproximadamente normal de la distribución de la puntuación total, que nos autorizará el uso de los programas de análisis de varianza. Asimismo hemos obtenido un valor tipificado del coeficiente de asimetría y curtosis dentro de los límites [-2,2] admitidos dentro de los límites de normalidad.

\section{Figura 1}

Distribución de frecuencias de la puntuación total.

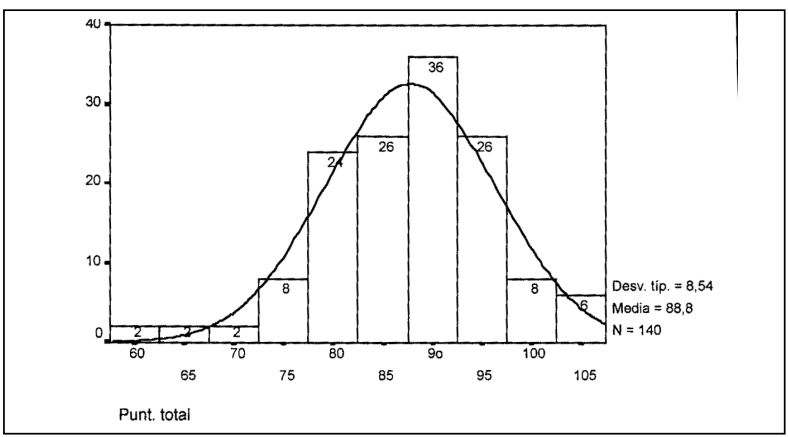

Relación entre las variables «grupo» y «género» y la actitud hacia la estadística

En la tabla III presentamos los resultados del análisis de varianza factorial con dos factores intersujetos: género (con dos niveles) y grupo (también con dos niveles: profesores en formación o en ejercicio). A la vista de los resultados obtenidos, observamos que sólo el de grupo tiene un efecto estadísticamente significativo sobre la puntuación total en la escala de actitudes, pero no sucede lo mismo con el de género ni la interacción entre ambos.

En la tabla IV presentamos las medias, desviaciones típicas e intervalos de confianza totales, según grupo y género, que nos ayudarán a completar el estudio.

De acuerdo con los resultados del análisis de varianza, no hay una gran diferencia en puntuaciones medias entre hombres y mujeres (sólo dos puntos de diferencia), lo que contradice los resultados de otras investigaciones anteriores, que indican que la actitud hacia la estadística es peor en el caso de las mujeres (Cazorla et al., 1998). Posiblemente, para los profesores que han participado en esta investigación, esta diferencia no sea tan acusada, puesto que ninguno de ellos ha tenido ocasión de estudiar estadística a fondo ni de utilizarla demasiado durante su trabajo profesional.

Respecto a la diferencia entre grupos, observamos una mejor actitud en los profesores en ejercicio, respecto a la que presentan los futuros profesores (una diferencia de 6 puntos), aunque, en realidad, la diferencia en puntuaciones no es importante. Al comparar las diferencias de los dos grupos en ítems aislados, obtuvimos mejores resultados para los profesores en ejercicio en los ítems $2,4,6,7,8,9,10,12,15$ y 22 , lo que indica que son más críticos con el uso de la estadística en los medios de comunicación, que le encuentran mayor utilidad para la comprensión del mundo de hoy, que le otorgan mayor valor para la formación general del ciudadano, que son más partidarios de incluirla en la enseñanza, que la encuentran más sencilla, comprensible, interesante y menos aburrida, y que suelen ayudar a otros compañeros con el tema. Los estudiantes superan a los profesores en ejercicio en los ítems 1 y 3 , por lo que confían más en el uso que se hace de la estadística.

\section{Influencia de las variables escolares en el grupo de profesores en formación}

En la tabla V mostramos los resultados del análisis factorial de varianza respecto a estudios previos (4 niveles) y especialidad (2 niveles).

No es significativa la especialidad pero si los estudios previos y la interacción entre ambos.

En la figura 2 presentamos la gráfica donde se analizan las medias combinadas por estudios previos y especialidad que completarán el estudio de la interacción de estas variables.

Observamos diferencias en actitud dentro de los que estudiaron estadística en magisterio, según se trate de la especialidad en educación infantil o en primaria, seguramente porque los segundos poseían mejores útiles matemáticos para comprender la materia y apreciar su utilidad.

Al analizar los ítems en que hubo una diferencia estadísticamente significativa, podemos concluir que aquellos individuos que no han estudiado nunca estadística son los que presentan actitudes más negativas, lo que nos confirma nuestro objetivo de potenciar la formación como una de las principales causas de mejora de la educación estadística. Aunque podemos observar actitudes positivas en el resto de colectivos, debido a factores que suponemos evolutivos y propios de la situación académica, el colectivo que la estudió en magisterio es el que se muestra con peores actitudes, debido al escaso tiempo dedicado al tema, que no permite mejorar el nivel de formación. También nos parece oportuno señalar las diferencias que presentan los que la estudiaron en COU, en aquellos ítems en que se pone de manifiesto la utilidad multidisciplinar de la materia debido, tal y como ya hemos argumentado anteriormente, al tipo de instrucción correspondiente a este nivel y donde el profesorado tiende a impartirla, más teórica que práctica. 
Tabla III

Análisis de varianza de la puntuación total en función de género y grupo.

\begin{tabular}{|l|c|c|c|c|c|}
\hline Fuente & Suma de cuadrados & Gl & Media cuadrática & F & Sig. \\
\hline Grupo & 676,44 & 1 & 676,44 & 10,15 & 0,00 \\
Género & 76,51 & 1 & 76,51 & 0,89 \\
Grupo *Género & 1,39 & 1 & 1,39 & 0,15 & 0,29 \\
Error & $9.061,99$ & 136 & 66,63 & 0,19 \\
Total & $1.113 .742,00$ & 140 & & & \\
\end{tabular}

$a$ calculado con alfa $=0,05$

Tabla IV

Medias, desviaciones típicas e intervalos de confianza.

\begin{tabular}{|l|l|c|c|c|c|}
\hline & & Media & $\begin{array}{c}\text { Errores } \\
\text { típicos }\end{array}$ & \multicolumn{2}{|c|}{ Intervalo de confianza al 95\% } \\
\hline \multirow{2}{*}{ Total } & & & & Límite inferior & Límite superior \\
Género & Hombre & 88,35 & 0,93 & 86,51 & 90,19 \\
& Mujer & 87,35 & 1,69 & 84,02 & 90,69 \\
\multirow{2}{*}{ Grupo } & Profesores en ejercicio & 89,35 & 0,79 & 87,78 & 90,92 \\
& Profesores en formación & 85,38 & 1,53 & 83,43 \\
\hline
\end{tabular}

Tabla V

Análisis de varianza de la puntuación total en función de especialidad y estudios previos.

\begin{tabular}{|l|c|c|c|c|c|c|}
\hline \multicolumn{1}{|c|}{ Fuente } & $\begin{array}{c}\text { Suma de } \\
\text { cuadrados }\end{array}$ & Gl & Media cuadrática & F & Sig. & $\begin{array}{c}\text { Potencia } \\
\text { observada }\end{array}$ \\
\hline Especialidad & 192,71 & 1 & 192,71 & 2,72 & 0,10 & 0,37 \\
Estudios previos & 764,33 & 4 & 191,08 & 2,70 & 0,04 & 0,72 \\
Especialidad* Estudios & 682,76 & 3 & 227,59 & 3,21 & 0,03 & 0,72 \\
Error & $4.602,17$ & 65 & 70,80 & & & \\
Total & 556.676 & 74 & & & \\
\end{tabular}

Figura 2

Medias según estudios previos y especialidad.

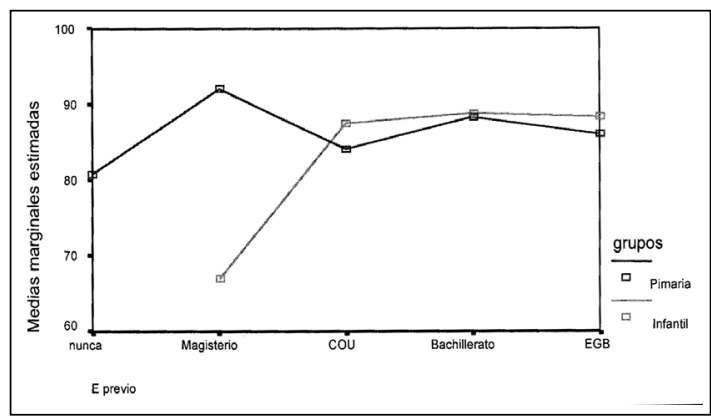


Influencia de las variables escolares en el grupo de profesores en ejercicio

Estudiamos la influencia de la especialidad y años de docencia para la submuestra de profesores en ejercicio. Puesto que la variable «años de docencia» es numérica, hemos utilizado el análisis de covarianza y hemos obtenido resultados estadísticamente no significativos para la especialidad. En cuanto a los años de docencia, el coeficiente de correlación $(r=-0,37)$ es significativo, aunque no muy intenso al nivel $\alpha=0,037$ y negativo, lo que apunta a la posibilidad de que la actitud global hacia la estadística se empeora con los años de enseñanza de la misma, posiblemente porque los profesores encuentran dificultades con el tema por parte de los alumnos o incluso en ellos mismos.

En el caso del colectivo de profesores del área de sociales, aparecía, en su cuestionario, la pregunta referida al uso de la estadística en clase y, al realizar el análisis de la varianza, cuyos resultados presentamos en la tabla VI, observamos que efectivamente esta variable es significativa, calculado con alfa $=0,05$.

Además, en la tabla VII, presentamos las medias, desviaciones típicas e intervalos de confianza totales según el grado de utilización, y resulta curioso como la media que aparece más elevada corresponde precisamente al colectivo de profesores del área de sociales, que no utiliza nunca la estadística en sus clases. Este resultado hay que interpretarlo con precaución, dado que la muestra es pequeña, aunque de todos modos nos aporta un dato para la reflexión.

Las otras dos medias se sitúan muy próximas entre sí, pero a bastante distancia de la anterior, lo que induce una vez más a la reflexión sobre la formación específica y didáctica que reciben los profesores actuales.

\section{CONCLUSIONES Y PERSPECTIVAS DE ESTU- DIO}

En primer lugar, los resultados obtenidos indican que las actitudes hacia la estadística de los profesores en formación y en ejercicio tienen características muy similares y, aunque la diferencia no sea muy elevada, encontramos una mayor puntuación media en los profesores en ejercicio, lo que nos induciría a pensar que la práctica docente mejora las actitudes.

Asimismo, nuestro estudio sugiere que estas actitudes son, en general, moderadas y positivas, con una puntuación de promedio global superior a la posición teórica de indiferencia. Mientras que las investigaciones de Auzmendi (1992) y Wilensky $(1995,1997)$ indican que los alumnos muestran actitudes negativas hacia la estadística, nuestro estudio con profesores se aparta de esta tendencia y está más en línea con los resultados de Matracci (2000) o Gil Flores (1999).

Tabla VI

Resultados del análisis de varianza de la puntuación total en función del uso de la estadística en clase.

\begin{tabular}{|l|c|c|c|c|c|c|}
\hline Fuente & Suma de cuadrados & Gl & $\begin{array}{c}\text { Media } \\
\text { cuadrática }\end{array}$ & F & Sig. & $\begin{array}{c}\text { Potencia } \\
\text { observada }\end{array}$ \\
\hline Uso de la estadística & 461,80 & 2 & 230,90 & 5,92 & 0,01 & 0,84 \\
Error & $1.209,96$ & 31 & 39,03 & & & \\
Total & $291.660,00$ & 34 & & & & \\
\hline
\end{tabular}

Tabla VII

Estadísticas de la puntuación total según uso de la estadística.

\begin{tabular}{|l|c|c|c|c|}
\hline & Media & Error típico & \multicolumn{2}{|c|}{ Intervalo de confianza al 95\% } \\
\hline Uso de la estadística & & & Límite inferior & Límite superior \\
Nunca & 107,00 & 4,42 & 97,99 & 116,01 \\
Algunas veces & 91,73 & 1,33 & 89,01 & 94,44 \\
Siempre & 90,80 & 1,98 & 86,77 & 94,83 \\
\hline
\end{tabular}


Debido al tamaño limitado de la muestra y a las características del instrumento utilizado, no se aporta información, en esta fase de la investigación, sobre los componentes diferenciados de las actitudes.

Por lo que se refiere al efecto de las variables personales y escolares sobre las actitudes hacia la estadística de los profesores en formación y en ejercicio, a la vista de los resultados obtenidos y de acuerdo con los resultados del análisis de varianza, se confirma, aunque sólo parcialmente, nuestra hipótesis 2 . Así, la variable «género» no es estadísticamente significativa, lo que contradice los resultados de otras investigaciones anteriores, como la de Cazorla y otros (1998), que indica que la actitud hacia la estadística es peor, en el caso de las mujeres.

Al analizar las diferencias entre especialidades en los profesores en formación, encontramos mejores puntuaciones en la especialidad de primaria, en comparación con la de infantil, tanto desde un punto de vista global como en ítems aislados. Ello es lógico debido a que el primer grupo recibe una formación algo más completa en el área de matemáticas.

En el caso de los estudios previos, las puntuaciones más bajas se presentan en los que nunca estudiaron estadística, confirmando nuestra teoría de que es preciso enseñar este tema en la formación de profesores si queremos mejorar sus actitudes y con ello incidir en que la estadística se incorpore realmente en el currículo de educación primaria. Más sorprendente es el hecho de no haber encontrado apenas diferencia entre las actitudes de los que han estudiado estadística únicamente en magisterio y los que nunca la han estudiado, pero esto se explica porque, en realidad, dentro de los estudios de magisterio, apenas se dedica un crédito (10 horas) al tema, lo que es claramente insuficiente.
Respecto a la variable «años de docencia en la materia» los resultados obtenidos apuntan a la idea de que la actitud global hacia la estadística empeora con los años de enseñanza de la misma, probablemente porque los profesores encuentran dificultades con el tema por parte de los alumnos o incluso en ellos mismos. Esto se ve, no sólo en el análisis de las puntuaciones globales, sino también de los ítems aislados. Es particularmente preocupante el hecho de que estos ítems se refieran a considerar la estadística de interés para la formación del alumno, la necesidad de enseñarla en la escuela y el deseo de eliminarla si fuera posible.

Finalmente, los resultados apuntan a que los profesores del área de sociales son los que menos han usado la estadística, los que mejores actitudes tienen hacia la materia, aunque en el estudio de las puntuaciones globales aparecen efectos significativos. Este resultado aparentemente sorprendente refleja, a nuestro entender, la dificultad de los profesores en ejercicio respecto al tema, al no contar con un asesoramiento adecuado desde los centros de profesores, por lo que respecta a la estadística y su enseñanza. Son bastantes los ítems aislados en que esta situación se reproduce y, a pesar de que por el tamaño limitado de la muestra no podemos generalizar estas conclusiones, cuando menos nos deben incitar a la reflexión.

A la vista de las anteriores conclusiones, la información obtenida, a pesar del tamaño limitado de la muestra, nos permite realizar una primera aproximación a las actitudes hacia la estadística en profesores en formación y profesores en ejercicio, contrastar diferentes hipótesis respecto al efecto de variables sobre las mismas y, sobre todo, nos aporta una información original, a la vez que abre un camino en el estudio de las actitudes de los profesores en formación en el ámbito de la estadística, que debe ser continuado en futuras investigaciones, puesto que el tema apenas ha sido tratado. 


\section{REFERENCIAS BIBLIOGRÁFICAS}

AIKEN, L. R. (1974). Two scales of attitude toward mathematics. Journal for Reseach in Mathematics Education, 5, pp. 67-71.

AUZMENDI, E. (1992). Las actitudes hacia la matemática estadística en las enseñanzas medias y universitarias. Bilbao: Mensajero.

CAZORLA, I. M., SILVA, C. B., DA VENDRAMINI, C. y BRITO, M. R. F. (1998). Adaptaçao e validaçao de uma escala de attitudes em relaçao à Estatística, en Actas de la Conferência Internacional: Experiências e Perspectivas do Ensino da Estatística, pp. 45-58. Florianópolis: Presta

ESTRADA, A. (2001). Evaluación de actitudes hacia la estadística, en Cardeñoso, J. M. et al., (ed.). Actas de las Jornadas de Investigación en el aula de Matemáticas. Atención a la diversidad, pp. 157-162. Departamento de Didáctica de la Matemática. Universidad de Granada.

ESTRADA, A. (2002). «Análisis de las actitudes y conocimientos estadísticos elementales en la formación del profesorado». Tesis doctoral. Universidad Autónoma de Barcelona.

GAIRÍN, J. (1987). Las actitudes en educación. Barcelona: PPU.

GAL, I. (2002). Adult's statistical literacy: Meaning, components, responsibilites. International Statistical Review, $70(1)$, pp. $1-52$.

GAL, I., GINSBURG, L. y SCHAU, C. (1997). Monitoring attitudes and beliefs in statistics education, en Gal. I. y Garfield, J. B. (ed.). The assessment challenge in statistics education, pp. 37-51. Voorburgo: IOS Press.

GATTUSO, L. y PANNONE, M. A. (2002). Teacher's training in a statistics teaching experiment, en Phillips, B. (ed.). Proceedings of the Sixth International Conference on Teaching of Statistics. Ciudad del Cabo: IASE. CD ROM.

GIL FLORES, J. (1999). Actitudes hacia la estadística. Incidencia de las variables sexo y formación previa. Revista Española de Pedagogía, 214, pp. 567-590.

GÓMEZ CHACÓN, I. M. (2000). Matemática emocional. Los afectos en el aprendizaje matemático. Madrid: Narcea.

LLINARES, S. (1994). El profesor de matemáticas. Conocimiento base para la enseñanza y desarrollo profesional, en Santaló, L., Llinares, S., Sánchez, V., Taibo, A. y GarcíaHoz, A. (eds). La enseñanza de las ciencias en la educacion intermedia, pp. 296-337. Madrid: Rialp Editores.

MASTRACCI, M. (2000). «Gli aspecti emotive nell'evolution dell'apprendimento della statistica e della sua valutazione.
Un caso di studio sugli studenti di SSA». Tesis de Laurea. Universidad La Sapienza de Roma.

McLEOD, D. B. (1992). Research on affect in mathematics education: A reconceptualization, en Grows, D. A. (ed.). Hanbook of research on mathematics teaching and learning pp. 575-596. Nueva York: MacMillam-NCTM.

MOORE, D. S. (1997). New pedagogy and new content: The case of statistics. International Statistical Review, 65(2), pp. 123-155.

MORALES, P. (1988). Medición de actitudes en psicología y educación. Universidad de Comillas. San Sebastián.

OSTERLIND, S. (1989). Constructing test items. Boston: Kluwer.

ROBERTS, D. M. y BILDERBACK, E. W. (1980). Reliability and validity of a statistics attitudes survey. Educational and Psychological Measurement, 40, pp. 235-238.

ROKEACH, M. (1968). Beliefs, attitudes and values. San Francisco: Jossey-Bass.

SCHAU, C., STEVENS, J., DAUPHINE, T. y DEL VECCHIO, A. (1995). The development and validation of the survey of attitudes towards statistics. Educational and Psychological Measurement, 55 (5), pp. 868-875.

SUYDAM, M. N. (1984). Research report: Attitudes toward mathematics. Arithmetic Teacher, 32, pp. 12-19.

TANUR, J. M. (1992). La Estadística: una guía de lo desconocido. Madrid: Alianza Editorial.

THORNDIKE, R. L. (1989). Psicometría aplicada. México: Limusa.

THOMPSON, A. G. (1992). Teachers' beliefs and conceptions: A synthesis of the research, en Grouws, D.A. (ed.). Handbook on Mathematics Teaching and Learnings, pp. 127-146. Nueva York: MacMillan.

WILENSKY, U. (1995). Learning probability through building computational models, en Carraher, D. y Meira, L. (eds.). Proceedings of the 19th PME Conference, 11, pp. 152-159. Brasil: Recife.

WILENSKY, U. (1997). What is normal anyway? Therapy for epistemological anxiety. Educational Studies in Mathematics, 33, pp. 171-202.

WISE, S. L. (1985). The development and validation of a scale measuring attitudes toward statistics. Educational and Psychological Measurement, 45, pp. 401-405. 